\title{
Pilates exercises improve low back pain and quality of life in patients with HTLV-1 virus: A randomized crossover clinical trial
}

Jaqueline Borges, DP ${ }^{a, c}$, Abrahão Fontes Baptista, PT, PhD ${ }^{a, b, c, *, 1}$, Nayara Santana, PT ${ }^{c}$, Israel Souza, AT d, Ramon A. Kruschewsky, MD, MsC e , Bernardo Galvão-Castro, MD, PhD ${ }^{a, e}$, Katia Nunes Sá, PT, PhD ${ }^{a, c, e, 1}$

\footnotetext{
a Escola Bahiana de Medicina e Saúde Pública, Post-graduation Program on Medicine and Human Health, Salvador, Bahia, Brazil

b Universidade Federal da Bahia, Biomorphology Department, Salvador, Bahia, Brazil

c Escola Bahiana de Medicina e Saúde Pública, Neuromusculoskeletal Dynamics Research Group, Salvador, Bahia, Brazil

${ }^{\mathrm{d}}$ Instituto Federal de Ciência e Tecnologia do Rio de Janeiro, Paracambi, Rio de Janeiro, Brazil

e Escola Bahiana de Medicina e Saúde Pública, Multidisciplinary Reference Center for HTLV and Human Retroviruses, Salvador, Bahia, Brazil
}

Received 3 October 2012; received in revised form 15 April 2013; accepted 5 May 2013

\section{KEYWORDS \\ HTLV-1; \\ Pilates exercises; \\ Low back pain; \\ Quality of life}

\begin{abstract}
Summary Background: Low back pain is highly prevalent in patients with HTLV-1. The effects of physical activity on this condition are not known, but postural misalignment and motor deficits are frequently present.

Objectives: To assess the effect of Pilates exercises on chronic low back pain in these patients, and its impact on quality of life.

Methods: A randomized crossover clinical trial was conducted, involving 22 patients from a reference center in Salvador, Bahia, Brazil. The VAS was used to evaluate the effect of Pilates on pain intensity and the SF-36 to assess its impact on quality of life.

Results: Our results provide evidence of positive effects on pain intensity and almost all domains of quality of life when patients followed the Pilates exercise program described.
\end{abstract}

\footnotetext{
* Corresponding author. Laboratório de Eletroestimulação Funcional, Departamento de Biomorfologia, Universidade Federal da Bahia, Av. Reitor Miguel Calmon, s/n - Vale do Canela, CEP: 40110-902 Salvador, Bahia, Brazil. Tel./fax: +55 7132838888.

E-mail address: abrahao.baptista@gmail.com (A.F. Baptista).

1 These authors contributed equally for this study.
} 
Conclusion: The Pilates method may be a useful tool in alleviating the symptoms of low back pain, and had a significant impact on quality of life in this sample of patients.

(c) 2013 Elsevier Ltd. All rights reserved.

\section{Introduction}

Human T-lymphotropic virus type 1 (HTLV-1) was the first human retrovirus identified (Poisez et al. 1980). HTLV-1 exists worldwide, with about 20 million people infected. This virus is endemic in certain areas of the world such as Africa, Japan, Malaysia, the Caribbean islands, Central America, and Latin America (Mueller et al., 1996). In Brazil, the city of Salvador has the highest prevalence of cases in the country (Dourado et al., 2003). About 5\% of infected individuals develop severe pathologies (Manns et al., 1999). Among the clinical alteration commonly observed, HTLV-1 associated myelopathy/tropical spastic paraparesis (HAM/ TSP), which is associated with HTLV-1 infection, interferes most with HTLV patients' quality of life, due to its neurological effects (Ribas and Melo, 2002).

HAM/TSP mainly affects females between the ages of 35 and 49 and progresses slowly, causing reductions in muscle strength, changes in the urinary system (retention or urinary incontinence), intestinal constipation, decreased libido and erectile dysfunction in males (Proietti et al., 2005). Ten years after the onset of HAM/TSP about $30 \%$ of patients develop paraplegia (Franzoi and Araújo, 2007). Individuals with HAM/TSP have biomechanical, functional and sensory abnormalities, characterized by decreased muscle strength of the pelvic girdle and lower limbs, gait disturbances, spasticity, muscle shortening and/or joint hypomobility, predisposing these individuals to postural abnormalities and generalized pain, which can affect their quality of life (Macêdo et al., 2002).

Low back pain is the most common type of pain in patients infected by the HTLV-1 (Netto and Brites, 2011), and may be related to muscle dysfunction, which is even more pronounced in the presence of spasticity (Gessain et al., 1985). Low back pain in these patients is generally treated with analgesics, but their prolonged use, due to the chronic nature of the pain, causes serious side effects. This has led to the search for alternative therapies that target the causes of the pain.

The Pilates method can improve physiological and psychological functions, as well as teach functional posture and appropriate motor patterns, and may be useful for these patients (Goldby et al., 2006; La Touche et al., 2008). This method of exercise is based on attention to the details of movement, and patterning of significant muscle groups, especially the core (powerhouse) muscles (Muscolino and Cipriani, 2004). Dysfunction of these muscles has been associated with LBP (Hodges and Richardson, 1996). Previous studies addressing the functional recovery of patients with other diseases that exhibit similar symptoms to those of HTLV-1 point to the benefits of specific therapeutic exercise programs (La Touche et al., 2008), suggesting that a program based on the Pilates method could be useful for patients with HTLV-1 associated back pain (Posadzki et al.,
2011). Therefore, the aim of this study was to assess the possible/potential therapeutic effect of the Pilates method on chronic low back pain in patients infected by the HTLV-1 and its impact on patients' quality of life.

\section{Methods}

A randomized crossover clinical trial was developed, involving patients infected by the HTLV-1 from the Integrative and Multidisciplinary Center for HTLV (CHTLV) of the Bahian School of Medicine and Public Health in Salvador, Bahia, Brazil. The following individuals were included in the study: 1) Individuals infected by the HTLV-1, who had reported low back pain for at least six months (felt everyday or almost everyday); 2) Age 18 or older and younger than $65 ; 3$ ) Able to understand the exam questions and proposed exercises; 4) Able to empty the bladder before each therapeutic session voluntarily or by catheterization. The following were considered exclusion criteria for the study: 1) Physical deformity; 2) Associated neurological deficits, unrelated to the viral disease; 3) Patients doing another physical activity; 4) Pregnant women.

The project was approved by the Research Ethics Committee of the Bahian School of Medicine and Public Health, under protocol number 083/2010. Signed consent was obtained from participants after they had been given verbal and written explanations of the study protocol, in accordance with the principles of Resolution 196/96 of the Brazilian National Board of Health.

The sample size calculation was based on an improvement of six points in the visual analogue scale, a standard deviation of four points, an alpha value of $5 \%$, and beta of $10 \%(1-\beta=90 \%)$. These parameters generated a sample size of nine subjects per group. We included two more subjects in each group to address potential dropout issues, which generated a sample size of 11 subjects per group.

Patients were selected by the CHTLV neurologist, and classified according to the WHO criteria for the presence of HTLV-1 infection, randomized by a table of random numbers and allocated into groups by a team member who did not participate in the assessment, treatment, or statistical analysis phases.

Pain intensity was considered the primary outcome of the study, and quality of life the secondary one. For pain evaluation, body maps were used to verify the location of the symptom, and the visual analogue scale (VAS) to measure its intensity varying from 0 (no pain) to 10 (worst possible pain). The simplified version of the SF- $36{ }^{\circledR}$ Health Survey was used to evaluate quality of life (Campolina et al., 2011). This survey consists of 11 questions and 36 items assessed in eight domains: physical functioning, physical role functioning, emotional role functioning, bodily pain, general health perceptions, vitality, mental 
health and social role functioning. A score that varies from 0 to 100 represents each domain.

Twenty-eight patients were invited to participate in the study. These patients were evaluated for eligibility in an initial interview by a trained team. After this interview, informed consent was obtained. Five patients did not meet the inclusion criteria and one patient did not attend the initial interview. A blinded member of the team assigned the 22 patients to two groups using a table of random numbers. Half of the patients (11) were placed into group A (Pilates-control), and started with Pilates method exercises immediately. Group B (Control-pilates) was made up of the other 11 patients, who continued their daily activities without any changes for 15 weeks, when they began to follow the Pilates program. After 30 sessions, the groups switched their activities. Blind and experienced examiners administered all evaluation procedures before the intervention, after 15 (when the switch was made) and 30 weeks (end of the study).

The exercise program based on the Pilates method consisted of one-hour sessions twice a week for a total of 30 classes taught by previously trained staff. On the first class of the week, the exercises were held on the Reformer and on the second class on the Cadillac and Mat-Pilates. The exercises are described bellow and illustrated on Fig. 2. The program was carried out in a standardized manner according to protocols developed by staff of the CHTLV of the Bahian School of Medicine and Public Health, from January to August, 2011. In the first classes five repetitions of each exercise were held. Repetitions were increased to six and eight from the 11th to 20th and from 21st to 30th class, respectively. Before starting the study protocol, two theoretical-practical orientation meetings were held with all participants to elucidate the methods principles (concentration, breathing, centering, flow, precision and control). The study protocol was developed to a slow and progressive evolution. It aimed to pelvic girdle stabilization, initially alone and later with girdle dissociation, mobilization and strengthening of spine and upper and lower extremities. For the strengthening exercises series, the equipments were set up with a series of springs listed bellow. As physical capacity enhanced, loads were changed (higher or lower) to increase challenge.

The training protocol

Reformer

Footwork series (Fig. 2A)

Equipment preparation: Two heavy springs or one medium and one heavy springs.

In supine position, starting with the feet on the bar, with knees and hips flexed, pushing the carriage up to the limit of the lower limbs active extension, then back to starting position.

\section{Footwork running/tendon stretch (Fig. 2B)}

Equipment preparation: Two or three medium springs.

In supine position, with the feet on the bar and knees straight, planti and dorsiflexing the ankles, stretching the calf. Then simulating a running movement, bending one knee, keeping the other extended, pushing the heel into the carriage, stretching the calf.
Hamstring series (Fig. 2C)

Equipment preparation: two medium springs.

In supine position, with upper limbs along the body, and straps on ankle joints, flexing and extending the hips while maintaining knee joints straight. Patients also performed the leg circles, a variation, where the handles were positioned on the thighs and the movements simulated the drawing of circles and figures of eight, mobilizing the hips.

Pelvic lift/bridge on bar (Fig. 2D)

Equipment preparation: two or three medium springs.

In supine position with hips and knees bent at approximately 70 degrees, shoulders supported on the shoulder pads and heels resting on the bar, making a bridge while holding the carriage still.

Stomach massage (Fig. 2E)

Equipment preparation: Two medium springs and low bar position.

Sitting on the reformer on deep C-curve position with feet on the bar, hips, knees and trunk flexed. Pushing the carriage, extending knees and hips and taking the trunk to a vertical position, moving the upper limbs straight, above the head, and coming back to the initial position. Patients who had good flexibility were asked to hold the bar while extending the knees and moving the carriage backward.

\section{Quadruped (Fig. 2F)}

Equipment preparation: one light spring.

In quadruped position, with the hands resting on the fixed side of the device. Knees should start with a flexion of approximately 70 degrees. Pulling the carriage away from the feet bar, until reaching an angle of $90^{\circ}$ of hips and knees, and coming back to the starting position.

\section{Standing series (Fig. 2G)}

Equipment preparation: One medium spring and a fixed platform.

In the standing position, with one foot on the platform and the other on the carriage. Pushing and pulling the carriage, abducting and adducting the hips.

\section{Cadillac}

Sit up (Fig. 2H)

Equipment preparation: two short and light springs attached to the upper position of the Cadillac.

In supine position, flexing the trunk, with knees extended, holding the contraction of the abdominal muscles.

\section{Side Kick Series (Fig. 2I)}

Equipment preparation: one light, long spring attached to the intermediate position of the Cadillac. Springs were 
used to provide assistance. The strap attached the spring to the upper thigh and progressed to upper leg.

On the side lying position, moving the upper lower limb (knee straight or flexed) forward and backward.

\section{Monkey (Fig. 2J)}

Equipment preparation: one heavy, long spring attached to the lower level of the Cadillac.

In supine position, with the back, but not the head supported by the mat, holding the bar with the hands and one foot. Pushing the bar extending the knee, and moving the trunk away from the mat.

\section{Cat/push down (Fig. 2K)}

Equipment preparation: Two light, short springs or one medium spring attached to the upper position of the Cadillac.

Kneeling in front of the bar, pushing the bar up and down, extending and flexing the trunk to a $\mathrm{C}$-curve position.

\section{Spine stretch (Fig. 2L)}

Equipment preparation: two light, short springs attached to the upper position of the Cadillac.

The patient should be seated on a proper Swiss ball, holding the bar, flexing and extend the trunk in a "diving" motion.

Mat pilates (not illustrated)

Arch of femur $90 / 90$

Equipment preparation: two light and short springs attached to the upper position of the Cadillac.

In supine position with the strap on the ankle knee joints, with $90^{\circ}$ of hips and knees, moving the hip and knee joints doing circles, figures of eight and cycling movements, uni- and bilaterally.

\section{Hamstring series (on foam roll) (not illustrated)}

Equipment preparation: One or two light, short springs, depending if the exercises were done uni- or bilaterally. The height was adjusted to the individual height, strength and flexibility of the patient.

In supine position, laying down on the roll, one foot on the floor and the other lifted by the spring, extending and flexing the hip, with knee straight.

\section{Analyses}

Analyses were made of the following treatmentsequence groups: Pilates-control and Control-pilates. To evaluate whether or not the subjects were adequately randomized into groups, the independent samples $t$-test was used. To evaluate the impact of the training program on the analyzed variables over the evaluation period, the linear mixed models for longitudinal data was used (Senn, 2002). Additionally, the correlated random effects model was used for the individuals evaluated, and the period used was a repeated effect in a model with correlated residuals within the random effects.
To analyze the effect of treatment, $p<0.05$ was used as the criterion for statistical significance. As for the carryover effect, $p<0.10$ was used as the criterion for statistical significance. The estimated treatment effects and their confidence intervals, as well as the tests for statistical significance, were performed using the MIXED procedure in Statistical Package for the Social Sciences (SPSS) version 17.0 for Windows.

\section{Results}

Twenty-eight patients diagnosed with HAM/TSP were evaluated for eligibility. Of these, six were excluded, five because they had sporadic pain and one because contact was lost. The final sample of 22 patients was made up of 16 women and six men, with a mean age of $48.73 \pm 10.07$ years, with a mean body mass index of $25.18 \pm 4.07$. During the study five patients dropped out, but the analyses included the 22 patients in the intent to treat model (Fig. 1). At baseline, the randomization technique was used to ensure verification of the compatibility of the groups (Table 1).

The behavior of pain intensity and quality of life are summarized in Table 1. There was significant reduction in pain intensity, as well as improvement in almost all domains of the SF-36 after the Pilates exercise protocol. In the control-pilates group, the parameters evaluated from the baseline to the first reevaluation after three months remained the same or deteriorated. When the group that served as the control in first phase began the exercise program, a significant response in almost all parameters with respect to the baseline was seen during the second reevaluation. The effects of time, gender, and sequence of treatment were insignificant. With regard to Emotional Role functioning, the effect of treatment was insignificant.

\section{Discussion}

Our results demonstrated that a Pilates exercise program had a positive impact on pain intensity and quality of life in patients with HTLV-1 associated low back pain. Improvement was seen in pain intensity as evaluated by the patients, as well as almost all the studied quality of life domains: physical functioning, physical role functioning, bodily pain, vitality, general health perceptions, social role functioning and mental health. However, no impact was found on emotional role functioning. This is the first study to evaluate the impact of the potential therapeutic effect of a Pilates exercise program on patients with a neurologic disease and high prevalence of LBP and lumbo-pelvic postural abnormalities.

Low back pain is known to be associated with muscle weakness (Bayramoglu et al., 2001), low endurance (Del Pozo-Cruz et al., 2012) and motor control dysfunction (Hodges and Richardson, 1996; Jull and Richardson, 2000) of the deep core muscles such as the transverse abdominis, multifidus, pelvic floor and diaphragm. As pain increases, postural control deficits increase in the same order (Tsao et al., 2008; Spiko and Kuczynski, 2012), and this may be a key factor in the maintenance of pain and disability.

Individuals with limited walking capacity are at higher risk of developing muscular and joint pain as the disease progresses, probably due to the greater physical exertion required to walk and perform everyday activities (Netto and 


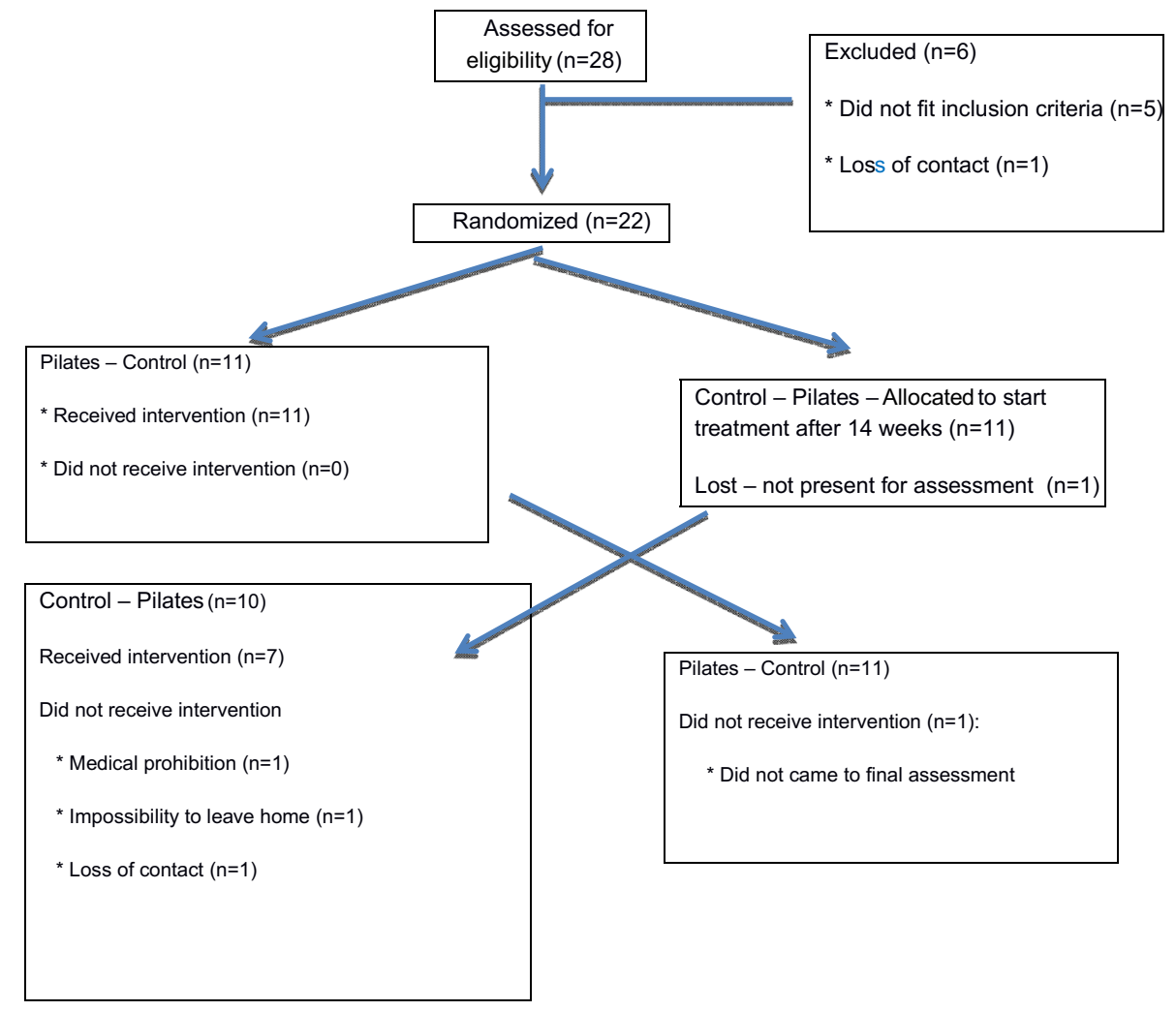

Figure 1 Flow diagram.

Brites, 2011). One of the main objectives of the Pilates method is to train these specific deep trunk stabilizing muscles (Posadzki et al., 2011; Gladwell et al., 2006; Lange et al., 2000) using static and dynamic resistance exercises, that may improve control of the sensorimotor system of the core, and provide limbs with more adequate mobility in daily physiological activities (Shinkle et al., 2012). As these properties improve, pain control may be achieved.

Posture is the result of the interaction between static and dynamic processes. Postural changes of individuals infected with the HTLV-1 cause damage to physical functioning and physical role functioning, possibly influenced by changes in proprioception, muscle weakness, biomechanical misalignments and postural instability, which Pilates exercises work to improve (Posadzki et al., 2011; Gladwell et al., 2006; Lange et al., 2000). Patients with HTLV-1 also present spasticity, which is associated with posture control deficits (Tsao et al., 2008; Sosnoff et al., 2010). Moreover, spasticity has a strong association with paresis (Wissel et al., 2013), which can contribute to obvious postural imbalances seen in these patients (Macêdo et al., 2002; Franzoi and Araújo, 2007). Successful strengthening of the lumbo-pelvic-hip complex is an effective way to improve static postural stability, as well as to increase abdominal pressure, reduce the load on the lumbar spine and adjacent structures, assist in the return to equilibrium, and decrease lower back pain (Goldby et al., 2006). Thus, the improvement in function and pain observed in our patients may be

Table 1 Study outcomes.

\begin{tabular}{|c|c|c|c|c|c|c|}
\hline \multirow[t]{2}{*}{ Variable } & \multicolumn{3}{|l|}{ Pilates-control } & \multicolumn{3}{|l|}{ Control-pilates } \\
\hline & Baseline & After pilates & $\begin{array}{l}\text { After the } \\
\text { waiting period }\end{array}$ & Baseline & $\begin{array}{l}\text { After the } \\
\text { waiting period }\end{array}$ & After pilates \\
\hline Pain intensity* & $7.18(2.35)$ & $3.45(2.54) \tau$ & $4.50(2.46)$ & $6.91(2.47)$ & $7.50(2.51)$ & $4.57(2.82) \tau$ \\
\hline Physical functioning & $23.18(14.88)$ & $41.82(20.16) \tau$ & $31.00(28.56)$ & $33.18(16.17)$ & $32.00(15.31)$ & $47.86(19.76) \tau$ \\
\hline Physical role functioning & $18.18(31.80)$ & $72.73(32.51) \tau$ & 32.50 (39.17) & $15.91(25.67)$ & $10.00(17.48)$ & $47.86(40.19) \tau$ \\
\hline Bodily pain & $31.82(14.75)$ & $60.64(20.11) \tau$ & $44.10(18.48)$ & $26.27(13.73)$ & $30.50(14.45)$ & $57.00(21.52) \tau$ \\
\hline General health perception & $46.36(24.13)$ & $52.73(25.73) \tau$ & $37.60(26.35)$ & $41.45(19.46)$ & $32.20(18.40)$ & $49.00(14.17) \tau$ \\
\hline Vitality & $23.18(18.20)$ & $56.36(22.70) \tau$ & $46.50(27.69)$ & $25.91(18.82)$ & $28.00(23.71)$ & $49.28(23.53) \tau$ \\
\hline Social role functioning & $46.59(30.15)$ & $69.32(20.43) \tau$ & $61.25(29.14)$ & $52.27(31.53)$ & $55.00(25.82)$ & $80.36(21.48) \tau$ \\
\hline Emotional role functioning & 33.36 (42.19) & $63.65(40.71) \Psi$ & $50.02(42.32)$ & $30.29(34.81)$ & 70.51 (24.59) & 76.17 (37.09) \\
\hline Mental health & $56.73(24.71)$ & $69.82(25.45) \tau$ & $61.60(27.85)$ & $46.78(27.15)$ & $54.00(22.25)$ & $69.71(17.26) \tau$ \\
\hline
\end{tabular}

*Variable where carry-over effect was significant for time of treatment $(P<0.005)$; Paired $t$-test $P<0.001(\tau)$ or $P<0.05(\Psi)$; values expressed as mean (SD). 


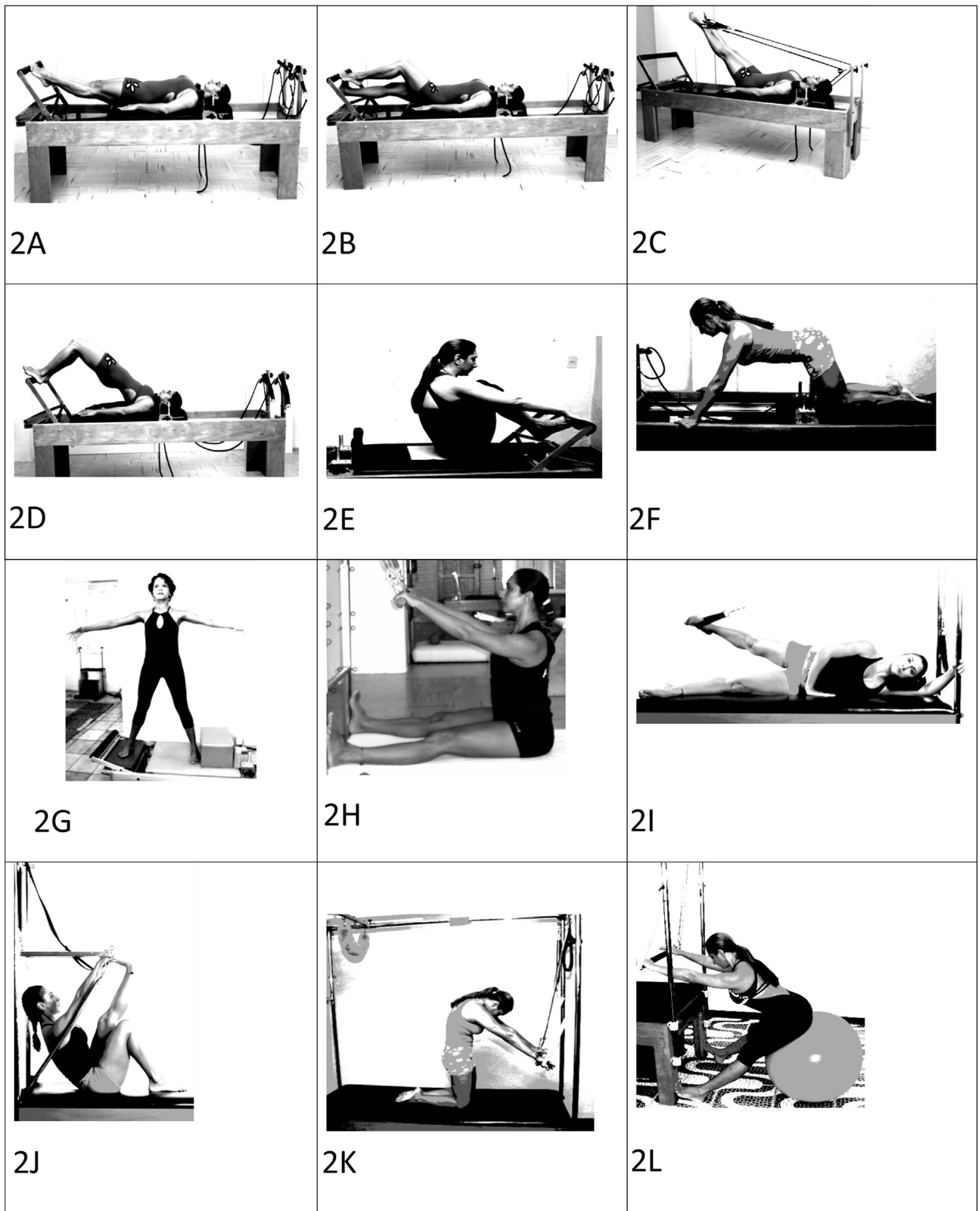

Figure 2 Protocol exercises: A - footwork series; B - footwork running; C - hamstring series; D - pelvic lift/bridge on bar; $E$ stomach massage; F - quadruped; G - standing series; $\mathrm{H}$ - sit up; I - Side Kick Series; J - monkey; K - cat/push down; L - spine stretch.

due to the influence of Pilates exercises on static posture (Kuo et al., 2009) and/or postural control (Emery et al., 2010), which still has to be verified.

Quality of life in patients with chronic low back pain is considerably impaired and often associated with a reduced sense of wellbeing (Nickel et al., 2002). Studies conducted with patients infected by the HTLV-1 show a negative impact of pain on the quality of life of these individuals, (Netto and Brites, 2011) indicating the need to include these measurements in the evaluation of therapeutic interventions. The 
overall findings of this study protocol using Pilates exercises is supported by the literature, demonstrating that a Pilates program may offer a low cost, safe alternative for the treatment of low back pain in this specific group of patients. Similar effects have been found in patients with chronic unspecific low back pain (La Touche et al., 2008), cancer (Eyigor et al., 2012) and fibromyalgia (Altan et al., 2009).

The limitations of this study include the lack of an objective measure of posture and muscle strength, which may explain some of the findings. In the statistical analysis of the results the group treatment sequence could be a source of bias. To be cautious, the effect of treatment was only analyzed in the first period and at baseline, having been dismissed at the second stage of the clinical trial.

To sum up, Pilates proved to be a useful tool to reduce self-reported LBP, which is the most common complaint of patients infected by the HTLV-1, and has a significant impact on their quality of life.

\section{References}

Altan, L., Korkmaz, N., Bingol, U., Gunay, B., 2009. Effect of pilates training on people with fibromyalgia syndrome: a pilot study. Archives of Physical Medicine and Rehabilitation 90 (12), 1983-1988.

Bayramoglu, M., Akman, M.N., Kilinç, S., Cetin, N., Yavuz, N., Ozker, R., 2001. Isokinetic measurement of trunk muscle strength in women with chronic low-back pain. American Journal of Physical Medicine and Rehabilitation 80 (9), 650-655.

Campolina, A.G., Bortoluzzo, A.B., Ferraz, M.B., Ciconelli, R.M., 2011. Validation of the Brazilian version of the generic sixdimensional short form quality of life questionnaire (SF-6D Brazil). Ciência e Saúde Coletiva 16 (7), 3103-3110.

Del Pozo-Cruz, B., Gusi, N., Adsura, J.C., Del Pozo-Cruz, J., Parraca, J.A., Hernandez-Mocholí, M., 2012. Musculoskeletal fitness and health-related quality of life characteristics among sedentary office workers affected by sub-acute, non-specific low back pain: a cross-sectional study. Physiotherapy 18. (Epub ahead of print).

Dourado, I., Alcantara, L.C., Barreto, M.L., Teixeira, M.G., GalvãoCastro, B., 2003. HTLV-1 in the general population of Salvador Brazil: a city with African ethnic and sociodemographic characteristics. Journal of Acquired and Immune Deficiency Syndromes 34 (5), 527-531, 15.

Emery, K., De Serres, S.J., McMillan, A., Côté, J.N., 2010. The effects of a Pilates training program on arm-trunk posture and movement. Clinical Biomechanics (Bristol, Avon) 25 (2), 124-130.

Eyigor, S., Karapolat, H., Yesil, H., Uslu, R., Durmaz, B., 2012. Effects of Pilates exercises on functional capacity, flexibility, fatigue, depression and quality of life in female breast cancer patients: a randomized controlled study. European Journal of Physical and Rehabilitation Medicine 46 (4), 481-487.

Franzoi, A.C., Araújo, A.Q., 2007. Disability and determinants of gait performance in tropical spastic paraparesis/HTLV-I associated myelopathy (HAM/TSP). Spinal Cord 45 (1), 64-68.

Gessain, A., Barin, F., Vernant, J.C., Gout, O., Maurs, L. Calender, A., de Thé, G., 1985. Antibodies to human T-lymphotropic vírus type-I in patients with tropical spastic paraparesis. Lancet 2 (8452), 407-410, 24.

Gladwell, V., Head, S., Haggar, M., Beneke, R., 2006. Does a program of Pilates improve chronic non-specific low back pain? Journal of Sports Rehabilitation 15, 338-350.

Goldby, L.J., Moore, A.P., Doust, J., Trew, M.E., 2006. A randomized controlled trial investigating the efficiency of musculoskeletal physiotherapy on chronic low back disorder. Spine (Phila Pa 1976) 31 (10), 1083-1093.

Hodges, P.W., Richardson, C.A., 1996. Inefficient muscular stabilization of the lumbar spine associated with low back pain. A motor control evaluation of transversus abdominais. Spine (Phila Pa) 21 (22), 2640-2650, 15.

Jull, G.A., Richardson, C.A., 2000. Motor control problems in patients with spinal pain: a new direction for therapeutic exercise. Journal of Manipulative Physiological Therapeutics 23 (2), 115-117.

Kuo, Y.L., Tully, E.A., Galea, M.P., (Phila Pa 1976), 2009. Sagittal spinal posture after Pilates-based exercise in healthy older adults. Spine 34 (10), 1046-1051, 1.

La Touche, R., Escalante, K., Linares, M.T., 2008. Treating nonspecific chronic low back pain through the Pilates method. Journal of Bodywork and Movement Therapy 12 (4), 364-370. Epub Feb 1.

Lange, C., Unnithan, V., Larkam, E., Latta, P.M., 2000. Maximizing the benefits of Pilates-inspired exercise for learning functional motor skills. Journal of Bodywork and Movement Therapies 4 (2), 99-108.

Macêdo, M.C., Andrade, A.S.F., Figueirôa, F.L.S., 2002. Avaliação das alterações biomecânicas, funcionais e sensoriais em pacientes portadores do HTLV-1 associado à HAM/TSP: Uma visão fisioterapêutica. Revista Brasileira de Neurologia e Psiquiatria 1 (6), 12-21.

Manns, A., Hisada, M., La Grenade, L., 1999. Human T-lymphotropic virus type I infection. Lancet 353 (9168), 1951-1958, 5.

Mueller, N., Okayama, A., Stuver, S., Tachibana, N., 1996. Findings from the Miyazaki Cohort study. Journal of Acquired Immune Deficiency Syndromes and Human Retrovirology 1 (13 Suppl), S2-S7.

Muscolino, J.E., Cipriani, S., 2004. Pilates and the powerhouse. Journal of Bodywork and Movement Therapies 8, 15-24.

Netto, E.C., Brites, C., 2011. Characteristics of chronic pain and its impact on quality of life of patients with HTLV-1-associated Myelopathy/Tropical Spastic Paraparesis (HAM/TSP). Clinical Journal of Pain 27 (2), 131-135.

Nickel, R., Egle, U.T., Rompe, J., Eysel, P., Hoffman, S.O., 2002. Somatisation predicts the outcome of treatment in patients with low back pain. Journal of Bone and Joint Surgery 84 (2), 189-195.

Posadzki, P., Lizis, P., Hagner-Derengowska, 2011. Pilates for low back pain: a systematic review. Complementary Therapy in Clinical Practice 17, 85-89.

Proietti, F.A., Carneiro-Proietti, A.B., Catalan-Soares, B.C., Murphy, E.L., 2005. Global epidemiology of HTLV-I infection and associated diseases. Oncogene 24 (39), 6058-6068, 5.

Ribas, J.G., Melo, G.C., 2002. Human T-cell lymphotropic vírus type 1(HTLV-1)-associated myelopathy. Revista da Sociedade Brasileira de Medicina Tropical 35 (4), 377-384.

Senn, S., 2002. Linear Mixed Models for Longitudinal Data. Springer-Verlag, New York.

Shinkle, J., Nesser, T.W., Demchak, T.J., McMannus, D.M., 2012. Effect of core strength on the measure of power in the extremities. Journal of Strength and Conditioning Research 26 (2), $373-380$.

Spiko, T., Kuczynski, M., 2012. Intensity of chronic pain modifies postural control in low back patients. European Journal of Pain. (Epub ahead of print).

Sosnoff, J.J., Shin, S., Motl, R.W., 2010. Multiple sclerosis and postural control: the role of spasticity. Archives of Physical Medicine and Rehabilitation 91 (1), 93-99.

Tsao, H., Galea, M.P., Hodges, P.W., 2008. Reorganization of the motor cortex is associated with postural control deficits in recurrent low back pain. Brain 131, 2161-2171.

Wissel, J., Manack, A., Brainin, M., 2013. Toward an epidemiology of poststroke spasticity. Neurology 80 (3 Suppl. 2), S13-S19, 15. 